doi: $10.13108 / 2021-13-3-113$

\title{
GROWTH OF ENTIRE FUNCTIONS OF EXPONENTIAL TYPE AND CHARACTERISTICS OF DISTRIBUTIONS OF POINTS ALONG STRAIGHT LINE IN COMPLEX PLANE
}

\author{
A.E. SALIMOVA, B.N. KHABIBULLIN
}

\begin{abstract}
According a classical Weierstrass-Hadamard-Lindelöf theorem, for each distribution of points with a finite upper density in the complex plane, there exists a non-zero entire function of exponential type vanishing on the these points with the multiplicity taken into account. In the beginning of 1960s, in a joint work by P. Malliavin and L.A. Rubel, the following problem was completely solved. Given two distributions of points on the positive half-line with finite upper densities, find relations between these distributions under which for each non-zero entire function of exponential type vanishing on one of the distributions, there exists a non-zero entire function of exponential type vanishing on the other distribution and having the absolute value not exceeding that of the first function. A complete solution of this problem going back to works by F. Carlson, T. Carleman, M. Cartwright, L. Schwartz, J.-P. Kahane and many others, was given in terms of so-called logarithmic characteristics of distributions of points, which are expressed via reciprocals to points in these distributions. In this paper we extend these results on complex distributions of the points separated from the imaginary axis by a pair of vertical angles of an arbitrary small opening; here we develop logarithmic characteristics for complex distributions of points. We consider three types of possible restrictions on the growth along the imaginary axis, very strict ones, as by P. Malliavin and L.A. Rubel, and less restrictive as in previous works by the second co-author. The main results are of a completed form and are formulated as criterions.
\end{abstract}

Keywords: entire function of exponential type, distribution of zeroes, growth of entire function, logarithmic characteristics and measures, Lindelöf condition.

Mathematics Subject Classification:30D15, 30D20

\section{INTRODUCTION}

1.1. Main results. For two point distributions $Z$ and $W$ of a finite upper density in the complex plane $\mathbb{C}$, the real axis of which is denoted by $\mathbb{R}$, we provide several versions of necessary and sufficient conditions for their location ensuring that for each entire function of exponential type $g \neq 0$ vanishing on $\mathbf{W}$, there exists an entire function of exponential type $f \neq 0$ vanishing on $\mathrm{Z}$ and obeying one of the following three conditions:

1) $|f(i y)| \leqslant|g(i y)|$ for all $y \in \mathbb{R}$, that is, everywhere on the imaginary axis $i \mathbb{R}$;

2) $\ln |f(i y)| \leqslant \ln |g(i y)|+o(|y|)$ as $y \rightarrow \pm \infty$;

A.E. Salimova, B.N. Khabibullin, Growth of entire functions of exponential type and charACTERISTICS OF POINTS DistribUtions ALONG STRAight LiNE IN COMPLEX PLANE.

(C) Salimova A.E., Khabibullin B.N. 2021.

The work by A.E. Salimova is supported by RFBR (grant no. 20-31-90074-Aspiranty). The work by B.N. Khabibullin is made in the framework of executing of Developing Program of Scientific Educational mathematical center of Privolzhsky Federal District (agreement no. 075-02-2021-1393).

Submitted May 5, 2021. 
3) for each number $\varepsilon>0$ there exists an entire function of exponential type $f \neq 0$ vanishing on $\mathbf{Z}$ and satisfying the inequality $\ln |f(i y)| \leqslant \ln |g(i y)|+\varepsilon|y|$ for all $y \in \mathbb{R} \backslash E$, where $E \subset \mathbb{R}$ is a set of a finite linear Lebesgue measure.

The present study is made in the framework of generalizing and developing a classical theorem by P. Malliavin, L.A. Rubel proved in 1960s [1, Thm. 4.1], in which there was considered only the case of location $\mathbf{Z} \subset \mathbb{R}^{+}$and $\mathbf{W} \subset \mathbb{R}^{+}$on the positive semi-axis $\mathbb{R}^{+} \subset \mathbb{R}$. The criterions are given in terms of majorizing special logarithmic characteristic and (sub)measures for $\mathbf{Z}$ by corresponding logarithmic characteristics and (sub)measures for W. At that, in the third option there are additional restrictions for $\mathbf{Z}$ and $\mathbf{W}$, while in the first and second options one has to assume for $\mathbf{Z}$ and $\mathbf{W}$ an asymptotic separating by angles from the imaginary axis, or that $W$ is completely contained in the right or left half-plane, or a Lindelöf kind condition for $\mathrm{W}$ along the imaginary axis $i \mathbb{R}$ on certain symmetricity of the imaginary parts of the reciprocals $1 / w$ for $w \in W$ of form

$$
\left|\sum_{\substack{\mathrm{w} \in \mathrm{w} \\ 1 \leqslant|\mathrm{w}| \leqslant r}} \operatorname{Im} \frac{1}{\mathrm{w}}\right|=O(1) \quad \text { as } r \rightarrow+\infty .
$$

We proceed to exact formulations.

1.2. Notation and convention. We shall often write one-point sets $\{x\}$ without curly brackets, that is, simply as $x$. For instance, for the set of natural numbers $\mathbb{N}:=\{1,2, \ldots\}$ we let $\mathbb{N}_{0}:=0 \cup \mathbb{N}=\{0,1,2, \ldots\}$. The set $\mathbb{R}$ of real numbers with standard ordering ( $\left.\leqslant, \sup / \inf \right)$, algebraic and topological structures is mostly considered as a real axis in the complex plane $\mathbb{C}$; $i \mathbb{R}$ is the imaginary axis, $\overline{\mathbb{R}}:=-\infty \cup \mathbb{R} \cup+\infty$ is the extended real line with two ends $\pm \infty \notin \mathbb{R}$, completed by inequalities $-\infty \leqslant x \leqslant+\infty$ for each $x \in \overline{\mathbb{R}}$ and equipped with a natural order topology, and $\overline{\mathbb{N}}:=\mathbb{N} \cup+\infty, \overline{\mathbb{N}}_{0}:=\mathbb{N}_{0} \cup+\infty$. Intervals in $\overline{\mathbb{R}}$ are connected subsets in $\overline{\mathbb{R}}$, like a segment $[a, b]:=\{x \in \overline{\mathbb{R}}: a \leqslant x \leqslant b\}$ with ends $a, b \in \overline{\mathbb{R}}$, where $[a, b]=\varnothing$ is the empty set as $a<b$, and also $(a, b]:=[a, b] \backslash a,[a, b):=[a, b] \backslash b$ and an open interval $(a, b):=(a, b] \cap[a, b)$. By definition, inf $\varnothing:=+\infty$ and $\sup \varnothing:=-\infty$. Right and left open half-plane are denoted respectively as $\mathbb{C}_{\mathrm{rh}}:=\{z \in \mathbb{C}: \operatorname{Re} z>0\}$ and $\mathbb{C}_{\mathrm{lh}}:=-\mathbb{C}_{\mathrm{rh}}$. By $\bar{D}(r):=\{z \in \mathbb{C}:|z| \leqslant r\}$ we denote a closed circle of radius $r \in \mathbb{R}^{+}$centered at the origin. For $x \in X \subset \overline{\mathbb{R}}$ we let $x^{+}:=\sup \{0, x\}, X^{+}:=\left\{x^{+}: x \in X\right\}$. To an extended scalar function $f: S \rightarrow \overline{\mathbb{R}}$ we associate its positive $f^{+}: s \underset{s \in S}{\longmapsto}(f(s))^{+} \in \overline{\mathbb{R}}^{+}$and negative part $f^{-}:=(-f)^{+}: S \rightarrow \overline{\mathbb{R}}^{+}$. If in the writing of the sum the upper limit is less than the lower one or the summing is made over an empty set, then this sum is let to be zero.

1.3. Points distribution in complex plane. To each distribution of points $Z=\left\{z_{j}\right\}$ in $\mathbb{C}$ consisting of points $z_{j} \in \mathbb{C}$ indexed by at most countably many indices $j$ we associate a counting measure [2, 0.1 .2$]$

$$
n_{\mathrm{Z}}: S \underset{S \subset \mathbb{C}}{\longmapsto} \sum_{\mathrm{z}_{\mathrm{j}} \in S} 1 \in \overline{\mathbb{N}}_{0}
$$

is the number of points $z_{j}$ in $S$. The same notation

$$
n_{\mathrm{Z}}: z \underset{z \in \mathbb{C}}{\longmapsto} n_{\mathrm{Z}}(z)=\sum_{\mathrm{z}_{\mathrm{j}}=z} 1 \in \overline{\mathbb{N}}_{0}
$$

is used also for the counting function of distribution of points $\mathbf{Z}$, and

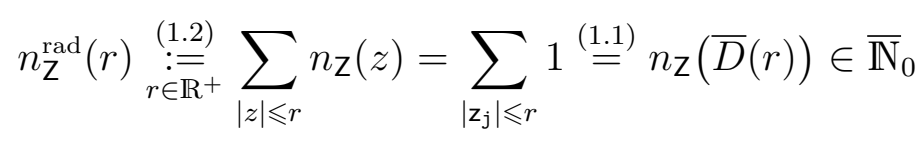

is a radial counting function for $\mathbf{Z}$ over closed circles $\bar{D}(r)$. 
Points distributions $\mathbf{Z}$ and $\mathbf{Z}^{\prime}$ coincides, or equal, which is written as $\mathbf{Z}=\mathbf{Z}^{\prime}$, if they have the same counting measures or functions $n_{\mathrm{Z}} \stackrel{1.1}{=} n_{\mathrm{Z}^{\prime}}$, while the inclusion $\mathrm{Z} \subset \mathrm{Z}^{\prime}$ means that the inequality $n_{\mathrm{Z}} \stackrel{1.2}{\leqslant} n_{\mathrm{Z}^{\prime}}$ holds true. A union $\mathrm{Z} \cup \mathrm{Z}^{\prime}$ is defined by the counting measure or the function $n_{\mathrm{Z} \cup Z^{\prime}}:=n_{\mathbf{Z}}+n_{\mathbf{Z}^{\prime}}$, and as $\mathbf{Z} \subset \mathbf{Z}^{\prime}$, a difference $\mathbf{Z}^{\prime} \backslash \mathbf{Z}$ is defined by the counting measure or the function $n_{\mathbf{Z}^{\prime} \mathbf{Z}}:=n_{\mathbf{Z}^{\prime}}-n_{\mathrm{Z}}$. A point $z \in \mathbb{C}$ belongs to $\mathbf{Z}$, that is, $z \in \mathbf{Z}$ if $n_{\mathbf{Z}}(z)>0$ for counting function $(1.2)$, and $z \notin \mathrm{Z}$ if $n_{\mathrm{Z}}(z)=0$. For $S \subset \mathbb{C}$ the intersection $\mathrm{Z} \cap S$ is defined by the restriction of $n_{\mathrm{Z}}$ to $S$, while the inclusion $\mathrm{Z} \subset S$ means that $\mathrm{Z}=\mathrm{Z} \cap S$.

An upper density (at order 1 ) of distribution of points $Z$ is denoted as [3]- 5 ]

$$
\overline{\mathrm{dns}}_{\mathrm{Z}}:=\limsup _{r \rightarrow+\infty} \frac{n_{\mathrm{Z}}^{\mathrm{rad}}(r)}{r} \in \overline{\mathrm{R}}^{+} \text {. }
$$

In what follows, a key role will be played by logarithmic characteristics for points distribution $\mathbb{C}$ introduced for positive distributions of points $\mathbf{Z} \subset \mathbb{R}^{+}$in a fundamental for our study paper by P. Malliavin, L.A. Rubel [1], see also a monograph by L.A. Rubel [6], and extended for arbitrary complex distributions of points $\mathrm{Z} \subset \mathbb{C}$ in works by B.N. Khabibullin [7]-[10], [2, 3.2].

We define right and left characteristic logarithms for $\mathbf{Z} \subset \mathbb{C}$ as

$$
\begin{aligned}
& l_{\mathrm{Z}}^{\mathrm{rh}}(r):=\sum_{\substack{0<\left|\mathrm{z}_{k}\right| \leqslant r \\
\mathrm{z}_{k} \in \mathbb{C}_{\mathrm{rh}}}} \operatorname{Re} \frac{1}{\mathrm{z}_{k}}=\sum_{0<\left|\mathrm{z}_{k}\right| \leqslant r} \operatorname{Re}^{+} \frac{1}{\mathrm{z}_{k}} \in \overline{\mathbb{R}}^{+}, \quad 0<r \leqslant+\infty, \\
& l_{\mathrm{Z}}^{\mathrm{lh}}(r):=\sum_{\substack{0<\left|\mathrm{z}_{k}\right| \leqslant r \\
\mathrm{z}_{k} \in \mathbb{C}_{\mathrm{lh}}}}-\operatorname{Re} \frac{1}{\mathrm{z}_{k}}=\sum_{0<\left|\mathrm{z}_{k}\right| \leqslant r} \operatorname{Re}^{-} \frac{1}{\mathrm{z}_{k}} \in \overline{\mathbb{R}}^{+}, \quad 0<r \leqslant+\infty,
\end{aligned}
$$

and also right and left logarithmic measures of intervals $(r, R] \subset \overline{\mathrm{R}}^{+}$:

$$
\begin{aligned}
& l_{\mathrm{Z}}^{\mathrm{rh}}(r, R) \stackrel{\sqrt[1.5]{:=}}{\mathrm{Z}_{\mathrm{Z}}^{\mathrm{rh}}}(R)-l_{\mathrm{Z}}^{\mathrm{rh}}(r) \in \overline{\mathrm{R}}^{+}, \quad 0<r<R \leqslant+\infty, \\
& l_{\mathrm{Z}}^{\mathrm{lh}}(r, R) \stackrel{\sqrt[1.6]{:=}}{=} l_{\mathrm{Z}}^{\mathrm{hh}}(R)-l_{\mathrm{Z}}^{\mathrm{lh}}(r) \in \overline{\mathbb{R}}^{+}, \quad 0<r<R \leqslant+\infty,
\end{aligned}
$$

which generate a logarithmic submeasure of intervals $(r, R] \subset \overline{\mathrm{R}}^{+}$for $\mathbf{Z}$

$$
l_{\mathrm{Z}}(r, R):=\max \left\{l_{\mathrm{Z}}^{\mathrm{lh}}(r, R), l_{\mathrm{Z}}^{\mathrm{rh}}(r, R)\right\} \in \overline{\mathbb{R}}^{+}, \quad 0<r<R \leqslant+\infty,
$$

where for $\mathbf{Z}=\varnothing$ by definition we let $l_{\varnothing}(r, R) \equiv 0$ for all $0<r<R \leqslant+\infty$.

1.4. Entire functions, known results and formulations of problems. A ring $\mathrm{Hol}(\mathbb{C})$ over $\mathbb{C}$ consists of all holomorphic functions on $\mathbb{C}$, that is $\mathrm{Hol}(\mathbb{C})$ is a ring of entire functions. By $\mathrm{Hol}_{*}(\mathbb{C}):=\{f \in \operatorname{Hol}(\mathbb{C}): f \neq 0\}$ we denote the set of all non-zero entire functions. By Zero $f$ we denote the distribution of all zeroes of an entire function $f \neq 0$ with a counting function $n_{\text {Zero }}$ in the sense of $(1.2)$, and at each point $z \in \mathbb{C}$ this counting function is equal to the multiplicity of the zero of the function $f$ at the point $z$. An entire function $f \neq 0$ on $\mathbb{C}$ vanishes on a distribution of points $\mathbf{Z}$, which is written as $f(\mathbf{Z})=0$, if $\mathbf{Z} \subset \mathbf{Z e r o}_{f}$.

An entire function $f$ is called an entire function of exponential type, which is shortly written as EFET, if its type (at order 1) is finite [3]-[5], [11, 2.1], [12, (1.1)]:

$$
\text { type }_{f}:=\limsup _{z \rightarrow \infty} \frac{\ln |f(z)|}{|z|} \text {. }
$$

Point distribution $\mathbf{Z}=\left\{\mathrm{z}_{\mathrm{j}}\right\} \subset \mathbb{C}$ generates an ideal [1], [6, Ch. 22], [12]

$$
I(\mathrm{Z}):=\{f \in \operatorname{Hol}(\mathbb{C}): f(\mathrm{Z})=0\} \subset \operatorname{Hol}(\mathbb{C})
$$


in the ring $\operatorname{Hol}(\mathbb{C})$ as well as an ideal in the ring of all EFET: $\mathrm{s}^{1}$

$$
I^{1}(\mathrm{Z}):=I(\mathrm{Z}) \cap\left\{f \in \operatorname{Hol}(\mathbb{C}): \text { type }_{f} \stackrel{1.10}{<}+\infty\right\},
$$

after excluding the zero function from these ideals we denoted these sets as

$$
I_{*}(\mathrm{Z}):=I(\mathrm{Z}) \cap \mathrm{Hol}_{*}(\mathbb{C}), \quad I_{*}^{1}(\mathrm{Z}):=I^{1}(\mathrm{Z}) \cap \mathrm{Hol}_{*}(\mathbb{C}) .
$$

Hereafter $\mathbf{Z} \subset \mathbb{C}, \mathbf{W}=\left\{\mathrm{w}_{\mathrm{j}}\right\} \subset \mathbb{C}$ are points distributions of finite upper density

$$
\overline{\mathrm{dns}}_{\mathrm{Z}}+\overline{\mathrm{dns}}_{\mathrm{W}}<+\infty .
$$

By mes we denote the linear Lebesgue measure of the Euclidean length on $\mathbb{R}$.

Theorem 1.1 ([7, Main thm.], [8, Main thm.], [2, Thm. 3.2.1]). If $\mathrm{W} \subset \mathbb{C}_{\mathrm{rh}}$ is located in the right half-plane, then the following three statements are equivalent:

I. For each function $g \in I_{*}^{1}(\mathrm{~W})$, for each $\varepsilon \in \mathbb{R}^{+} \backslash 0$ there exist a function $f \in I_{*}^{1}(\mathrm{Z})$ and a Borel set $E \subset \mathbb{R}$, for which

$$
\ln |f(i y)| \leqslant \ln |g(i y)|+\varepsilon|y| \quad \text { for all } y \in \mathbb{R} \backslash E \text { and mes } E<+\infty \text {. }
$$

II. For each $\varepsilon \in \mathbb{R}^{+} \backslash 0$ there exist a pair of functions $g \in I_{*}^{1}(\mathrm{~W})$ with Zero $g \cap \mathbb{C}_{\mathrm{rh}}=\mathrm{W}$ and $f \in I_{*}^{1}(\mathrm{Z})$, as well as a Borel set $E \subset \mathbb{R}$, for which (1.12) holds.

III. For each $\varepsilon \in \mathbb{R}^{+} \backslash 0$ there exists a number $C \in \mathbb{R}^{+}$, for which

$$
l_{\mathrm{Z}}(r, R) \leqslant l_{\mathrm{W}}(r, R)+\varepsilon \ln \frac{R}{r}+C \quad \text { for all } 0<r<R<+\infty .
$$

In [9, (0.2)], a distribution of points $\mathbf{Z}=\left\{\mathbf{z}_{\mathbf{j}}\right\}$ is called separated (by angles) from $i \mathbb{R}$ if

$$
\left|\operatorname{Re} \mathbf{z}_{j}\right| \geqslant d\left|z_{j}\right| \text { for all } \mathbf{j} \text { and for some number } d>0 \text {. }
$$

Geometrically (1.14) condition means that all non-zero points in Z are located outside nonempty open vertical angles containing $i \mathbb{R} \backslash 0$. A distribution of points $\mathbf{Z}$ is asymptotically separated by angles from $i \mathbb{R}$ if [12, (1.2)]

$$
\left(\liminf _{j \rightarrow \infty} \frac{\left|\operatorname{Re} z_{j}\right|}{\left|z_{j}\right|}>0\right) \Longleftrightarrow\left(\limsup _{j \rightarrow \infty} \frac{\left|\operatorname{Im} z_{j}\right|}{\left|z_{j}\right|}<1\right) .
$$

Geometrically, the pair of equivalent restrictions (1.15) means that there exists a pair of nonempty open vertical angles containing $i \mathbb{R} \backslash 0$, for which the points $\mathbf{z}_{\mathbf{j}}$ are located outside this pair of angles for all $j$ except finitely many of them.

Theorem 1.2 ([12, Main thm.]). Assume that under condition $(1.11)$ both $\mathrm{Z} \subset \mathbb{C}$ and $\mathrm{W} \subset$ $\mathbb{C}_{\mathrm{rh}}$ are asymptotically separated by angles from $i \mathbb{R}$ in the sense of $(1.15)$. Then the following three statements are equivalent:

I. For each function $g \in I_{*}^{1}(\mathrm{~W})$ with the zeros distribution asymptotically separated by angles from $i \mathbb{R}$ there exists a function $f \in I_{*}^{1}(\mathrm{Z})$ obeying the condition

$$
\ln |f(i y)| \leqslant \ln |g(i y)|+o(|y|) \quad \text { as } y \rightarrow+\infty .
$$

II. There exists a pair $g \in I_{*}^{1}(\mathrm{~W})$ with $\mathrm{Zero}_{g} \cap \mathbb{C}_{\mathrm{rh}}=\mathrm{W}$ and $f \in I_{*}^{1}(\mathrm{Z})$ satisfying (1.16).

III. There exist $C \in \mathbb{R}^{+}$and a bounded function $d: \mathbb{R}^{+} \rightarrow \mathbb{R}^{+}$with $d(x) \underset{x \rightarrow+\infty}{=} o(1)$, for which the inequalities hold:

$$
l_{\mathrm{Z}}(r, R) \leqslant l_{\mathrm{W}}(r, R)+d(R) \ln \frac{R}{r}+C \quad \text { for all } 0<r<R<+\infty .
$$

\footnotetext{
${ }^{1}$ In [1] and [6] the ideal $I^{1}(\mathrm{Z})$ is respectively denoted as $\mathcal{F}(\mathrm{Z})$ and $F(\mathrm{Z})$.
} 
A motivating Theorems 1.1 and 1.2 result is a joint one by P. Malliavin, L.A. Rubel obtained in the beginning of 1960 only for distributions of points located only on the positive semi-axis $\mathbb{R}^{+}$, which are obviously separated by angles from the imaginary axis in the sense of (1.14).

Theorem 1.3 ([1, Thm. 4.1], [6, Ch. 22]). If under (1.11) both $\mathrm{Z} \subset \mathbb{R}^{+}$and $\mathrm{W} \subset \mathbb{R}^{+}$are located on the positive semi-axis, then the following three statements are equivalent:

I. For each function $g \in I_{*}^{1}(\mathrm{~W})$ there exists a function $f \in I_{*}^{1}(\mathrm{Z})$ obeying the restriction

$$
|f(i y)| \leqslant|g(i y)| \quad \text { for all } y \in \mathbb{R} \text {. }
$$

II. There exists a pair $g \in I_{*}^{1}(\mathrm{~W})$ with Zero $_{g} \cap \mathbb{C}_{\mathrm{rh}}=\mathrm{W}$ and $f \in I_{*}^{1}(\mathrm{Z})$ satisfying (1.18).

III. There exist $C \in \mathbb{R}^{+}$, for which

$$
l_{\mathrm{Z}}(r, R) \leqslant l_{\mathrm{W}}(r, R)+C \quad \text { for all } 0<r<R<+\infty .
$$

Our paper is aimed on the following three problems.

1. Extension of Malliavin-Rubel theorem 1.3 from distribution of positive points to distributions of complex points $Z \subset \mathbb{C}$ and $W \subset \mathbb{C}_{\mathrm{rh}}$ asymptotically separated by angles from the imaginary axis $i \mathbb{R}$; see Theorem 2.1 in Section 2 .

2. The total omitting of the condition on location of $\mathbf{W}$ in the right half-plane in Theorem 1.1 under some reformulation of statement II Theorem 4.1 Section 4 .

3. Replacement of location of $\mathrm{W}$ in the right half-plane $\mathbb{C}_{\mathrm{rh}}$ in Theorems 1.2 and 2.1 by the condition

$$
\sup _{r \geqslant 1}\left|\sum_{1<\left|w_{j}\right| \leqslant r} \operatorname{Im} \frac{1}{w_{j}}\right|<+\infty
$$

in Theorems 4.2 and 4.3 in Section 4 with some changes in intermediate statements II. Condition 1.20 obviously covers all distributions of real points $\mathrm{W} \subset \mathbb{R}$ and points distributions symmetric with respect to the real axis or the origin.

A key role in the proofs of all theorems in Section 3 is played by Theorem 3.2 in Section 3 on relations between logarithmic (sub)measures fro distributions of points and various versions of Lindelöf condition (3.1), 3.2) and (3.3) of genus 1 for distribution of points on $\mathbb{C}$.

\section{Extension of MALliavin-Rubel theOREM on Distributions of COMPlex Points}

Theorem 2.1. Under condition (1.11), for all $\mathrm{Z} \subset \mathbb{C}$ and $\mathrm{W} \subset \mathbb{C}_{\mathrm{rh}}$ asymptotically separated by angles from $i \mathbb{R}$ in the sense of (1.15), statements $I I I I$ in Theorem 1.3 are equivalent.

Proof. In order to prove the implication $\mathrm{I} \Longrightarrow \mathrm{II}$, it is sufficient to construct a non-zerom EFET $g$ with Zero $_{g} \cap \mathbb{C}_{\text {rh }}=$ W. As such function, we can choose EFET $g$ as an even canonical Hadamard-Weierstrass product [3]-[5]:

$$
g(z) \underset{z \in \mathbb{C}}{:=} \prod_{j}\left(1-\frac{z^{2}}{\mathbf{w}_{j}^{2}}\right) .
$$

In order to prove the implication $\mathrm{II} \Longrightarrow \mathrm{III}$ in notation

$$
J_{i \mathbb{R}}(r, R ; \ln |f|):=\frac{1}{2 \pi} \int_{r}^{R} \frac{\ln |f(i y) f(-i y)|}{y^{2}} \mathrm{~d} y, \quad 0<r<R<+\infty,
$$

for entire functions $f$ we shall employ the following statement.

Lemma 2.1 ([8, (1.3)], [9, (0.4)], [13, Prop. 4.1, (4.19)]). For each fixed number $r_{0}>0$ and for each EFET $f \neq 0$ the relation holds:

$$
\sup _{r_{0} \leqslant r<R<+\infty} \max \left\{\left|J_{i \mathbb{R}}(r, R ; \ln |f|)-l_{\text {Zero }_{f}}^{\mathrm{rh}}(r, R)\right|,\left|J_{i \mathbb{R}}(r, R ; \ln |f|)-l_{\text {Zero }_{f}}^{\operatorname{lh}_{1}}(r, R)\right|\right\}<+\infty .
$$


We take the logarithm of and integrating of inequality $(1.18)$ in statement II of MalliavinRubel theorem and then we integrate it over the segment $[r, R]$ and divide by $y^{2}$ as in (2.1). This gives the inequalities

$$
J_{i \mathbb{R}}(r, R ; \ln |f|) \leqslant J_{i \mathbb{R}}(r, R ; \ln |g|) \quad \text { for all } \quad r_{0} \leqslant r<R<+\infty .
$$

By Lemma 2.1 applied to EFET $f \neq 0$, for some number $C_{f} \in \mathbb{R}^{+}$we obtain:

$$
l_{Z}(r, R) \stackrel{\sqrt[1.9]{\leqslant}}{\leqslant} l_{\text {Zero }_{f}}(r, R) \stackrel{2.2}{\leqslant} J_{i \mathbb{R}}(r, R ; \ln |f|)+C_{f} \stackrel{2.3 p}{\leqslant} J_{i \mathbb{R}}(r, R ; \ln |g|)+C_{f}
$$

for each $r_{0} \leqslant r<R<+\infty$. Again by Lemma 2.1 but applied to to EFET $g \neq 0$, we can continue this chain of inequalities with some number $C_{g} \in \mathbb{R}^{+}$as follows:

$$
l_{\mathrm{Z}}(r, R) \leqslant J_{i \mathbb{R}}(r, R ; \ln |g|)+C_{f} \stackrel{2.2}{\leqslant} l_{\text {Zerog }^{\mathrm{rh}}}^{\mathrm{rh}}(r, R)+C_{g}+C_{f} \quad \text { for all } \quad r_{0} \leqslant r<R<+\infty .
$$

Definition (1.5) of the right logarithmic measure and condition Zero $\cap \cap \mathbb{C}_{\mathrm{rh}}=\mathrm{W} \subset \mathbb{C}_{\mathrm{rh}}$ of statement II imply that the right hand side of the above relation reads as

$$
l_{\mathrm{W}}^{\mathrm{rh}}(r, R)+C_{g}+C_{f}=l_{\mathrm{W}}(r, R)+C_{g}+C_{f} .
$$

Hence, according to definitions (1.7), (1.8) and 1.9$)$, for the constant $C:=C_{g}+C_{f}$ independent of $r>r_{0}$ and $R>r$, for sufficiently small $r_{0}>0$ we can consider all $0<r<R<+\infty$ and this gives desired inequalities $(1.19)$ and statement III.

To prove the implication $[\mathrm{III} \Longrightarrow \mathbb{I}$, we make use of the following theorem.

Theorem 2.2 ([9, Main thm.]). Let $g \neq 0$ be an EFET and the distributions of zeroes Zero $g$ and the distribution of points $\mathbf{Z}=\left\{\mathbf{z}_{\mathrm{j}}\right\}$ are separated by angles from $i \mathbb{R}$ in the sense of (1.14). Then there exists an EFET $f \neq 0$ vanishing on $\mathbf{Z}$ and obeying (1.18) if and only if there exists a number $M \in \mathbb{R}$ such that

$$
l_{Z}(r, R) \leqslant J_{i \mathbb{R}}(r, R ; \ln |g|)+M \quad \text { for all } \quad 1 \leqslant r<R<+\infty .
$$

Suppose that under the assumptions of Statement III of Theorem 2.1, the assumptions of Theorem 2.2 hold as well. Then for EFET $g \neq 0$ with $\mathrm{W} \subset$ Zero $_{g}$, by Lemma 2.1 there exists a number $C_{g} \in \mathbb{R}^{+}$, for which

$$
l_{\mathrm{Z}}(r, R) \stackrel{\sqrt[1.19]{\leqslant}}{\leqslant} l_{\mathrm{W}}(r, R)+C \leqslant l_{\text {Zero }_{g}}(r, R)+C \stackrel{2.2)}{\leqslant} J_{i \mathbb{R}}(r, R ; \ln |g|)+C_{g}+C
$$

for all $r_{0} \leqslant r<R<+\infty$. Hence, for $r_{0}:=1$ we obtain (2.5) and by the sufficiency in Theorem 2.2 there exists an EFET $f \neq 0$ with $f(Z)=0$ satisfying (1.18).

Let the points distributions $\mathrm{Z}$ and Zero $_{g} \supset \mathrm{W}$ be just asymptotically separated by angles from $i \mathbb{R}$ in the sense of $(1.15)$. We can always choose a finite distribution of points $Z_{0} \subset Z$ and a finite distribution of points $G_{0} \subset$ Zero $_{g}$ such that $Z_{\infty}:=Z \backslash Z_{0}$ and $G_{\infty}:=$ Zero $_{g} \backslash G_{0}$ are separated by angles from $i \mathbb{R}$ in the sense of (1.14). At that, in view of the finiteness of distribution of points $\mathrm{G}_{0}$, according to the definitions of logarithmic functions of intervals (1.5) - 1.6 and (1.7)-1.9), there exists a number $C_{0} \in \mathbb{R}^{+}$, for which $l_{\mathrm{G}_{0}}(r, R) \leqslant C_{0}$ for all $0<r<R<+\infty$ and this yields:

$$
\begin{aligned}
l_{\mathrm{Z}_{\infty}}(r, R) & \leqslant l_{\mathrm{Z}}(r, R) \stackrel{1.19}{\leqslant} l_{\mathrm{W}}(r, R)+C \leqslant l_{\text {Zero }_{g}}(r, R)+C \stackrel{1.9 p}{\leqslant} l_{\mathrm{G}_{\infty}}(r, R)+l_{\mathrm{G}_{0}}(r, R)+C \\
& \leqslant l_{\mathrm{G}_{\infty}}(r, R)+C_{0}+C \quad \text { for all } \quad 0<r<R<+\infty .
\end{aligned}
$$

We consider an EFET $g_{\infty}:=g / g_{0} \neq 0$, where $g_{0}$ is some polynomial with zeroes distribution $\mathrm{G}_{0}$ and $g_{\infty}\left(\mathrm{G}_{\infty}\right)=0$. By the already proven version of the implication III $\Longrightarrow$ I for $g_{\infty}$ as $g$ and $\mathrm{G}_{\infty}$ as $\mathrm{W}$, in view of $(2.6)$, there exists an EFET $f_{\infty} \neq 0$ with $f_{\infty}\left(\mathrm{Z}_{\infty}\right)=0$ obeying condition (1.18) in the form $\left|f_{\infty}(i y)\right| \leqslant\left|g_{\infty}(i y)\right|$ for all $y \in \mathbb{R}$ and by our constructions, this implies:

$$
\left|\left(g_{0} f_{\infty}\right)(i y)\right| \leqslant\left|\left(g_{0} g_{\infty}\right)(i y)\right|=|g(i y)| \text { for all } y \in \mathbb{R} \quad \text { and } \quad\left(g_{0} f_{\infty}\right)\left(\mathbf{Z}_{\infty}\right)=0 \text {. }
$$


Let the number of points in $\mathrm{Z}_{0}$ be equal to $N$, that is, $N \stackrel{1.1}{=} n_{\mathrm{Z}_{0}}(\mathbb{C})$, and $f_{0}$ be some polynomial of degree $N$ with zeroes distribution $Z_{0}$. Then for a sufficiently small number $a>0$ the absolute value restriction of the function

$$
f_{a}(z): \underset{z \in \mathbb{C}}{=} a f_{0}(z)\left(\frac{\sin i z}{z}\right)^{N}
$$

to $i \mathbb{R}$ is bounded by one and by $(2.7)$ for the EFET $f:=f_{a} g_{0} f_{\infty} \neq 0$ vanishing on $\mathbf{Z}=\mathbf{Z}_{0} \cup \mathbf{Z}_{\infty}$ we have:

$$
|f(i y)|=\left|\left(f_{a} g_{0} f_{\infty}\right)(i y)\right| \stackrel{2.7}{\leqslant}|g(i y)|
$$

for all $y \in \mathbb{R}$ and this completes the proof of the implication $\mathrm{III} \Longrightarrow \mathbb{1}$ and Theorem 2.1.

Remark 2.1. By analyzing the proofs of Theorems 1.1 and 1.2. as well as of Theorem 2.1 we see that in the proof of the implications III $\Longrightarrow 1$ in each of these theorems the location of the distribution of points $\mathrm{W}$ exactly in the right half-plane $\mathbb{C}_{\mathrm{rh}}$ is not used. Thus, the condition $\mathrm{W} \subset$ $\mathbb{C}_{\mathrm{rh}}$ in Theorems 1.1, 1.2 and 2.1 is employed only in the proof of the implications $I \Longrightarrow I I \Longrightarrow I I I$. It is clear the condition $\mathrm{W} \subset \mathbb{C}_{\mathrm{rh}}$ in these theorems can be replaced by the location of $\mathrm{W} \subset \mathbb{C}_{\mathrm{h}}$ in the left half-plane by means of the mirror symmetry with respect to $i \mathbb{R}$.

\section{LINDELÖF CONDITION OF KIND 1}

Point distribution $\mathbf{Z}=\left\{\mathrm{z}_{\mathrm{j}}\right\}_{\mathrm{j} \in \mathrm{J}}$ satisfies Lindelöf condition (of kind 1) if

$$
\sup _{r \geqslant 1}\left|\sum_{1<\left|z_{j}\right| \leqslant r} \frac{1}{z_{j}}\right|<+\infty ;
$$

satisfies R-Lindelöf condition (of kind 1) if

$$
\sup _{r \geqslant 1}\left|\sum_{1<\left|z_{j}\right| \leqslant r} \operatorname{Re} \frac{1}{z_{j}}\right|<+\infty ;
$$

satisfies $i \mathbb{R}$-Lindlöf condition (of kind 1 ) if (see 1.20)

$$
\sup _{r \geqslant 1}\left|\sum_{1<\left|z_{j}\right| \leqslant r} \operatorname{Im} \frac{1}{z_{j}}\right|<+\infty .
$$

A special role of Lindelöf condition (3.1) of kind 1 is due to Hadamard-Lindelöf theorem.

Theorem 3.1 ([4], [5], 3, 2.10]). If $f \neq 0$ is EFET, then the zeroes distribution Zero f $_{\text {has }}$ a finite upper density and satisfies Lindelöf condition (3.1). Vice versa, if a distribution of points $\mathbf{Z}$ is of a finite upper density and satisfies Lindelöf condition (3.1), then there exists an EFET $f \neq 0$ with Zero $_{f}=\mathbf{Z}$.

The next follows immediately definitions (3.1), (3.2) and (3.3).

Proposition 3.1. Let $\mathrm{Z} \subset \mathbb{C}$ be a distribution of complex points.

[L1] Z satisfies Lindelöf condition (3.1) if and only if Z satisfies simultaneously $\mathbb{R}$-Lindelöf condition (3.2) and $i \mathbb{R}$-Lindelöf condition (3.3).

[L2] The following three statements are equivalent:

(i) Z satisfies $\mathbb{R}$-Lindelöf condition (3.2);

(ii) for right and left logarithmic measures (1.7) and (1.8) the relation holds:

$$
\sup _{1 \leqslant r<R<+\infty}\left|l_{\mathrm{Z}}^{\mathrm{rh}}(r, R)-l_{\mathrm{Z}}^{\mathrm{lh}}(r, R)\right|<+\infty ;
$$

(iii) for logarithmic submeasure (1.9) the relation holds:

$$
\sup _{1 \leqslant r<R<+\infty}\left(\left|l_{\mathrm{Z}}^{\operatorname{lh}}(r, R)-l_{\mathrm{Z}}(r, R)\right|+\left|l_{\mathrm{Z}}(r, R)-l_{\mathrm{Z}}^{\mathrm{rh}}(r, R)\right|\right)<+\infty ;
$$


(iv) the rotation $i \mathrm{Z}:=\left\{i \mathbf{z}_{\mathbf{j}}\right\} \subset \mathbb{C}$ by the angle $\frac{\pi}{2}$ satisfies $i \mathbb{R}$-Lindelöf condition (3.3).

Point distribution $Z$ is separated (with respect to the distance) if there exists a number $d>0$ such that the distance between each two points in $\mathbf{Z}$ is at least $d$.

In the proof of the next three theorems we shall employ the following theorem.

Theorem 3.2. Let $\mathrm{Z} \subset \mathbb{C}$ be a distribution of points of a finite upper density. Then there exist separated distribution of real points $X \subset \mathbb{R}$ and $Y \subset \mathbb{R}$ having finite upper densities such that the union $\mathrm{Z} \cup \mathrm{X}$ satisfies $\mathbb{R}$-Lindelöf condition (3.2), the union $\mathrm{Z} \cup i \mathrm{Y}$ satisfies $i \mathbb{R}$-Lindelöf condition (3.3) and

$$
\begin{aligned}
& \sup _{1 \leqslant r<R<+\infty}\left(l_{\mathrm{Z} \cup \mathrm{X}}(r, R)-l_{\mathrm{Z}}(r, R)\right)<+\infty, \\
& \sup _{1 \leqslant r<R<+\infty}\left(l_{(i \mathrm{Z}) \cup \mathrm{Y}}(r, R)-l_{i \mathrm{Z}}(r, R)\right)<+\infty .
\end{aligned}
$$

At that, the union $\mathrm{Z} \cup \mathrm{X} \cup i \mathrm{Y}$ satisfies Lindelöf condition (3.1.

Proof. We consider a binary sequence $r_{k}:=2^{k}$ with $k \in \mathbb{N}_{0}$.

In order to construct distributions of points $\mathrm{X}=\left\{\mathrm{x}_{\mathrm{j}}\right\} \subset \mathbb{R}$, for each $k \in \mathbb{N}_{0}$ we make the following choice of the intervals $I_{k}^{ \pm}$and points $\mathrm{x}_{\mathrm{j}} \in I_{k}^{ \pm}$forming $\mathrm{X}$ :

[+] If $l_{\mathrm{Z}}^{\mathrm{rh}}\left(r_{k}, r_{k+1}\right) \leqslant l_{\mathrm{Z}}^{\mathrm{lh}}\left(r_{k}, r_{k+1}\right)$, then we let $I_{k}^{+}:=\left(r_{k}, r_{k+1}\right] \subset \mathbb{R}^{+}$. It is obvious that we can choose a finite subset in $N_{k} \in \mathbb{N}$ of mutually disjoint points $\mathrm{x}_{j} \in I_{k}^{+}$, which includes the point $r_{k+1}$ and partitions $I_{k}^{+}$into subintervals of equal lengths $\left(r_{k+1}-r_{k}\right) / N_{k}$ such that

$$
\begin{aligned}
l_{\mathrm{Z}}\left(r_{k}, r_{k+1}\right) & \stackrel{1.9}{=} l_{\mathrm{Z}}^{\mathrm{lh}}\left(r_{k}, r_{k+1}\right) \leqslant l_{\mathrm{Z}}^{\mathrm{rh}}\left(r_{k}, r_{k+1}\right)+\sum_{\mathrm{x}_{\mathrm{j}} \in I_{k}^{+}} \frac{1}{\mathrm{x}_{\mathrm{j}}} \\
& \leqslant l_{\mathrm{Z}}^{\mathrm{lh}}\left(r_{k}, r_{k+1}\right)+\frac{1}{r_{k}} \stackrel{1.9}{=} l_{\mathrm{Z}}\left(r_{k}, r_{k+1}\right)+\frac{1}{r_{k}} .
\end{aligned}
$$

[-] If $l_{\mathrm{Z}}^{\mathrm{lh}}\left(r_{k}, r_{k+1}\right)<l_{\mathrm{Z}}^{\mathrm{rh}}\left(r_{k}, r_{k+1}\right)$, then we let $I_{k}^{-}:=\left[-r_{k+1},-r_{k}\right) \subset-\mathbb{R}^{+}$, where we choose a finite subset in $N_{k} \in \mathbb{N}_{0}$ of mutually disjoint points $\mathrm{x}_{\mathrm{j}} \in I_{k}^{-}$including the point $-r_{k+1}$ and partitioning $I_{k}^{-}$into subintervals of equal length $\left(r_{k+1}-r_{k}\right) / N_{k}$ such that

$$
\begin{aligned}
l_{\mathrm{Z}}\left(r_{k}, r_{k+1}\right) & \stackrel{1.9 \mathrm{l}}{=} l_{\mathrm{Z}}^{\mathrm{rh}}\left(r_{k}, r_{k+1}\right) \leqslant l_{\mathrm{Z}}^{\mathrm{lh}}\left(r_{k}, r_{k+1}\right)+\sum_{\mathrm{x}_{\mathrm{j}} \in I_{k}^{-}} \frac{1}{-\mathrm{x}_{\mathrm{j}}} \\
& \leqslant l_{\mathrm{Z}}^{\mathrm{rh}}\left(r_{k}, r_{k+1}\right)+\frac{1}{r_{k}} \stackrel{1.9}{=} l_{\mathrm{Z}}\left(r_{k}, r_{k+1}\right)+\frac{1}{r_{k}} .
\end{aligned}
$$

First we are going to show that the distribution of points $X$ has a finite upper density. By construction, the upper bounds in (3.8) and (3.9) with the concluding identity imply:

$$
N_{k} \frac{1}{r_{k+1}} \leqslant \sum_{\mathrm{x}_{\mathrm{j}} \in I_{k}^{ \pm}} \frac{1}{\left|\mathrm{x}_{\mathrm{j}}\right|} \leqslant l_{\mathrm{Z}}\left(r_{k}, r_{k+1}\right)+\frac{1}{r_{k}} \stackrel{1.1)}{\leqslant} \int_{\bar{D}\left(r_{k+1}\right) \backslash \bar{D}\left(r_{k}\right)}\left|\operatorname{Re} \frac{1}{z}\right| \mathrm{d} n_{\mathrm{Z}}(z)+\frac{1}{r_{k}},
$$

where $n_{\mathrm{Z}}$ is a counting measure in (1.1). Passing to the radial counting function (1.3), we can continue inequalities as

$$
N_{k} \leqslant r_{k+1} \int_{r_{k}}^{r_{k+1}} \frac{1}{t} \mathrm{~d} n_{\mathrm{Z}}^{\mathrm{rad}}(t)+2 \leqslant r_{k+1} \frac{1}{r_{k}} \int_{r_{k}}^{r_{k+1}} \mathrm{~d} n_{\mathrm{Z}}^{\mathrm{rad}}(t)+2 \leqslant 2\left(n_{\mathrm{Z}}^{\mathrm{rad}}\left(r_{k+1}\right)-n_{\mathrm{Z}}^{\mathrm{rad}}\left(r_{k}\right)+1\right),
$$

and by summing this over $k$ we get the inequalities

$$
n_{\mathrm{X}}^{\mathrm{rad}}\left(r_{n+1}\right) \leqslant \sum_{k=0}^{k=n} 2\left(n_{\mathrm{Z}}^{\mathrm{rad}}\left(r_{k+1}\right)-n_{\mathrm{Z}}^{\mathrm{rad}}\left(r_{k}\right)+1\right) \leqslant 2 n_{\mathrm{Z}}^{\mathrm{rad}}\left(r_{n+1}\right)+2(n+1) \quad \text { for all } n \in \mathbb{N} \text {. }
$$


Hence, for each $n \in \mathbb{N}$ and for all $r \in\left(r_{n}, r_{n+1}\right]$ we obtain

$$
\frac{n_{\mathrm{X}}^{\mathrm{rad}}(r)}{r} \leqslant 2 \frac{n_{\mathrm{X}}^{\mathrm{rad}}\left(r_{n+1}\right)}{r_{n+1}} \leqslant 4 \frac{n_{\mathrm{Z}}^{\mathrm{rad}}\left(r_{n+1}\right)}{r_{n+1}}+4 \frac{n+1}{2^{n+1}}=O(1) \quad \text { as } \quad n \rightarrow \infty \quad \text { and } \quad r \rightarrow+\infty,
$$

and this proves that the upper density of the distribution of points $\mathrm{X}$ is finite.

Moreover, it follows from (3.10) and the finiteness of the upper density of $\mathbf{Z}$ that there exists $D \in \mathbb{R}^{+} \backslash 0$, for which $N_{k} \leqslant 2 n_{\mathrm{Z}}^{\mathrm{rad}}\left(r_{k+1}\right)+2 \leqslant D r_{k+1}$ for all $k \in \mathbb{N}_{0}$. Therefore,

$$
\frac{r_{k+1}-r_{k}}{N_{k}} \geqslant \frac{r_{k+1}-r_{k}}{D r_{k+1}}=\frac{1}{2 D} \text { for all } k \in \mathbb{N} .
$$

By constructions $[ \pm]$ this means that the distribution of points $X$ is separated.

Let us show that the union $\mathrm{Z} \cup \mathrm{X}$ satisfies condition (3.6).

As $n<N$, by construction [+] of a part of distribution of points $\mathrm{X}$ located on the positive semi-axis $\mathbb{R}^{+}$, the additivity of right (1.7) and left (1.8) logarithmic measures and the middle inequality in 3.8 imply

$$
\begin{aligned}
l_{\mathrm{Z} \cup \mathrm{X}}^{\mathrm{rh}}\left(r_{n}, r_{N}\right) & \stackrel{\sqrt{1.7})}{=} \sum_{k=n}^{N-1} l_{\mathrm{Z} \cup \mathrm{X}}^{\mathrm{rh}}\left(r_{k}, r_{k+1}\right) \stackrel{\sqrt[3.8]{\leqslant}}{\leqslant} \sum_{k=n}^{N-1}\left(l_{\mathrm{Z}}^{\mathrm{lh}}\left(r_{k}, r_{k+1}\right)+\frac{1}{r_{k}}\right) \\
& =\sum_{k=n}^{N-1} l_{\mathrm{Z}}^{\mathrm{hh}}\left(r_{k}, r_{k+1}\right)+\sum_{k=n}^{N-1} \frac{1}{r_{k}} \stackrel{1.8)}{=} l_{\mathrm{Z}}^{\mathrm{lh}}\left(r_{n}, r_{N}\right)+\sum_{k=n}^{N-1} \frac{1}{2^{k}} \stackrel{1.9)}{\leqslant} l_{\mathrm{Z}}\left(r_{n}, r_{N}\right)+2 .
\end{aligned}
$$

In the same way, by construction [-] of the part of distribution of points $\mathrm{X}$ located on the negative semi-axis $-\mathbb{R}^{+}$, the additivity of left (1.8) and right (1.7) logarithmic measures and the middle inequality (3.9) imply

$$
\begin{aligned}
l_{\mathrm{Z} \cup \mathrm{X}}^{\mathrm{lh}}\left(r_{n}, r_{N}\right) & \stackrel{\sqrt[1.8]{=}}{=} \sum_{k=n}^{N-1} l_{\mathrm{Z} \cup \mathrm{X}}^{\mathrm{lh}}\left(r_{k}, r_{k+1}\right) \stackrel{\sqrt[3.9]{\leqslant}}{\leqslant} \sum_{k=n}^{N-1}\left(l_{\mathrm{Z}}^{\mathrm{rh}}\left(r_{k}, r_{k+1}\right)+\frac{1}{r_{k}}\right) \\
& =\sum_{k=n}^{N-1} l_{\mathrm{Z}}^{\mathrm{rh}}\left(r_{k}, r_{k+1}\right)+\sum_{k=n}^{N-1} \frac{1}{r_{k}} \stackrel{1.7 \mathrm{f}}{=} l_{\mathrm{Z}}^{\mathrm{rh}}\left(r_{n}, r_{N}\right)+\sum_{k=n}^{N-1} \frac{1}{2^{k}} \stackrel{1.9}{\leqslant} l_{\mathrm{Z}}\left(r_{n}, r_{N}\right)+2 .
\end{aligned}
$$

By (3.11)-3.12 and definition $(1.9)$ of the logarithmic submeasure we find:

$$
l_{\mathrm{Z} \cup \mathrm{X}}\left(r_{n}, r_{N}\right) \stackrel{[1.9)}{=} \max \left\{l_{\mathrm{Z} \cup \mathrm{Ih}}^{\mathrm{lh}}\left(r_{n}, r_{N}\right), l_{\mathrm{Z} \cup \mathrm{XX}}^{\mathrm{rh}}\left(r_{n}, r_{N}\right)\right\} \leqslant l_{\mathrm{Z}}\left(r_{n}, r_{N}\right)+2 .
$$

Hence, for all $n \leqslant N$, for all $r_{n}<r \leqslant r_{n+1}$ and $r_{N}<R \leqslant r_{N+1}$ we obtain:

$$
\begin{aligned}
l_{\mathrm{Z}}(r, R) & \leqslant l_{\mathrm{Z} \cup \mathrm{X}}(r, R) \leqslant l_{\mathrm{Z} \cup \mathrm{X}}\left(r, r_{n+1}\right)+l_{\mathrm{Z} \cup \mathrm{X}}\left(r_{n+1}, r_{N}\right)+l_{\mathrm{Z} \cup \mathrm{X}}\left(r_{N}, r\right) \\
& \stackrel{3.13}{\leqslant} \frac{1}{r_{n}} n_{\mathrm{Z} \cup \mathrm{rad}}^{\mathrm{rad}}\left(r_{n+1}\right)+\left(l_{\mathrm{Z}}\left(r_{n+1}, r_{N}\right)+2\right)+\frac{1}{r_{N}} n_{\mathrm{Z} \cup \mathrm{X}}^{\mathrm{rad}}\left(r_{N+1}\right) \\
& \leqslant l_{\mathrm{Z}}(r, R)+\left(2+2 \frac{n_{\mathrm{Z} \cup \mathrm{r}}^{\mathrm{rad}}\left(r_{n+1}\right)}{r_{n+1}}+2 \frac{n_{\mathrm{Z} \cup \mathrm{rad}}\left(r_{N+1}\right)}{r_{N+1}}\right) .
\end{aligned}
$$

In view of the proven finiteness of the upper density of the distribution of points $\mathrm{X}$, the latter quantity in the brackets is bounded with respect to all $0 \leqslant n \leqslant N<+\infty$ and this proves (3.6).

Finally, let us confirm that the union $Z \cup X$ satisfies $\mathbb{R}$-Lindelöf condition (3.2).

Consructions $[ \pm]$ show that the intervals $I_{k}^{+} \subset \mathbb{R}^{+}$and $-I_{k}^{-} \subset \mathbb{R}^{+}$are disjoint and cover entire ray $[1,+\infty) \subset \mathbb{R}^{+}$.

In case $[+]$, in view of identities

$$
l_{\mathrm{Z}}^{\mathrm{rh}}\left(r_{k}, r_{k+1}\right)+\sum_{\mathrm{x}_{\mathrm{j}} \in I_{k}^{+}} \frac{1}{\mathrm{x}_{\mathrm{j}}}=l_{\mathrm{Z} \cup \mathrm{X}}^{\mathrm{rh}}\left(r_{k}, r_{k+1}\right) \quad \text { for all intervals } \quad\left(r_{k}, r_{k+1}\right]=I_{k}^{+} \subset \mathbb{R}^{+},
$$


by the middle inequality (3.8) we obtain:

$$
0 \leqslant l_{\mathrm{Z} \cup \mathrm{XX}}^{\mathrm{rh}}\left(r_{k}, r_{k+1}\right)-l_{\mathrm{Z}}^{\mathrm{lh}}\left(r_{k}, r_{k+1}\right) \leqslant \frac{1}{r_{k}}=\frac{1}{2^{k}},
$$

$\operatorname{abd} l_{\mathbf{Z}}^{\operatorname{lh}}\left(r_{k}, r_{k+1}\right)=l_{\mathrm{Z} \cup \mathrm{X}}^{\mathrm{lh}}\left(r_{k}, r_{k+1}\right)$ since in no points of distributions of points $\mathbf{X}$ were chosen in the opposite interval $-I_{k}^{+} \subset-\mathbb{R}^{+}$in construction $[+]$. Thus,

$$
0 \leqslant l_{\mathrm{Z} \cup \mathrm{rh}}^{\mathrm{rh}}\left(r_{k}, r_{k+1}\right)-l_{\mathrm{Z} \cup \mathrm{h}}^{\mathrm{lh}}\left(r_{k}, r_{k+1}\right) \leqslant \frac{1}{2^{k}} \quad \text { for all intervals } \quad\left(r_{k}, r_{k+1}\right]=I_{k}^{+} \subset \mathbb{R}^{+} .
$$

In case $[-]$, in view of identities

$$
l_{\mathrm{Z}}^{\mathrm{lh}}\left(r_{k}, r_{k+1}\right)+\sum_{\mathrm{x}_{\mathrm{j}} \in I_{k}^{+}} \frac{1}{-\mathrm{x}_{\mathrm{j}}}=l_{\mathrm{Z} \cup \mathrm{x}}^{\mathrm{lh}}\left(r_{k}, r_{k+1}\right), \quad \text { for all intervals } \quad\left(r_{k}, r_{k+1}\right]=-I_{k}^{-} \subset \mathbb{R}^{+},
$$

by the middle inequality in $(3.9)$ we obtain:

$$
0 \leqslant l_{\mathrm{Z} \cup \mathrm{X}}^{\mathrm{lh}}\left(r_{k}, r_{k+1}\right)-l_{\mathrm{Z}}^{\mathrm{rh}}\left(r_{k}, r_{k+1}\right) \leqslant \frac{1}{r_{k}}=\frac{1}{2^{k}},
$$

and $l_{\mathrm{Z}}^{\mathrm{rh}}\left(r_{k}, r_{k+1}\right)=l_{\mathrm{Z} \cup \mathrm{X}}^{\mathrm{rh}}\left(r_{k}, r_{k+1}\right)$ since no points for the distribution of points $\mathrm{X}$ was chosen in the opposite interval $-I_{k}^{-} \subset \mathbb{R}^{+}$in construction [-]. Thus,

$$
0 \leqslant l_{\mathrm{Z} \cup \mathrm{XX}}^{\mathrm{lh}}\left(r_{k}, r_{k+1}\right)-l_{\mathrm{Z} \cup \mathrm{xh}}^{\mathrm{rh}}\left(r_{k}, r_{k+1}\right) \leqslant \frac{1}{2^{k}} \quad \text { for all intervals } \quad\left(r_{k}, r_{k+1}\right]=-I_{k}^{-} \subset \mathbb{R}^{+} .
$$

As $r \in\left(r_{n}, r_{n+1}\right]$, we sum over $k \leqslant n$ and on the base of (3.14) and (3.15) we get:

$$
\begin{aligned}
& \left|l_{\mathrm{Z} \cup \mathrm{X}}^{\mathrm{lh}}(1, r)-l_{\mathrm{Z} \cup \mathrm{X}}^{\mathrm{rh}}(1, r)\right| \leqslant \sum_{k=0}^{n-1}\left|l_{\mathrm{Z} \cup \mathrm{X}}^{\mathrm{lh}}\left(r_{k}, r_{k+1}\right)-l_{\mathrm{Z} \cup \mathrm{XX}}^{\mathrm{rh}}\left(r_{k}, r_{k+1}\right)\right|+\left|l_{\mathrm{Z} \cup \mathrm{l}}^{\mathrm{lh}}\left(r_{n}, r\right)\right| \\
& \stackrel{3.14}{\leqslant}, 3.150 \sum_{k=0}^{n-1} \frac{1}{2^{k}}+\frac{1}{r_{n}} n_{\mathrm{Z} \cup \mathrm{X}}^{\mathrm{rad}}\left(r_{k+1}\right) \leqslant 2+2 \frac{n_{\mathrm{Z} \cup \mathrm{X}}^{\mathrm{rad}}\left(r_{k+1}\right)}{r_{n+1}}=O(1)
\end{aligned}
$$

for all $n \in \mathbb{N}$ and $r \in\left(r_{n}, r_{n+1}\right]$. By the equivalence (ii) $\Longleftrightarrow$ (i) of part L2 in Proposition 3.1 and by relation (3.4), this means that the distribution of points $\mathrm{Z} \cup \mathrm{X}$ satisfies $\mathrm{R}$-Lindelöf condition (3.2).

The existence of the distributions of points $Y$ with required properties including (3.7) follows immediately from the proven part of Theorem 3.2 for $\mathbf{X}$ after the rotation of $i \mathrm{Z}$ by the angle $\frac{\pi}{2}$ in view of the equivalence (i) $\Longleftrightarrow$ (iv) in part [L2] of Proposition 3.1. Lindelöf condition (3.1) for the union $\mathrm{Z} \cup \mathrm{X} \cup i \mathrm{Y}$ is implied part [L1] of proposition 3.1.

Corollary 3.1. Let a distribution of points $\mathrm{W} \subset \mathbb{C}$ possesses a finite upper density. Then: (a) There exists an EFET $g \neq 0$ with properties $g(\mathrm{~W})=0$ and

$$
\sup _{1 \leqslant r<R<+\infty}\left(l_{\text {Zerog }_{g}}(r, R)-l_{\mathrm{W}}(r, R)\right)<+\infty .
$$

(b) If the distribution of points $\mathrm{W}$ is asymptotically separated by angles from $i \mathbb{R}$ in the sense of (1.15) and satisfies $i \mathbb{R}$-Lindelof condition (1.20), then the EFET $g \neq 0$ satisfying simultaneously two properties of $\mathrm{W} \subset$ Zero $_{g}$ and (3.16) can be chosen with an asymptotically separated by angles from $i \mathbb{R}$ zeroes distribution Zero $_{g}$.

Proof. By Theorem 3.2, there exist distributions of points $\mathrm{X} \subset \mathbb{R}$ and $i \mathrm{Y} \subset i \mathbb{R}$ of a finite upper density, for which $\mathbf{W} \cup \mathrm{X} \cup i \mathrm{Y}$ satisfies Lindelöf condition (3.1) and (3.6) holds with $\mathrm{W}$ instead of Z. By Hadamard-Lindelöf theorem 3.1, there exists an EFET $g \neq 0$ with zeroes distribution Zero $_{g}=\mathrm{W} \cup \mathrm{X} \cup i \mathrm{Y}$ vanishing on $\mathrm{W}$, for which by (3.6) with $\mathrm{W}$ instead of $\mathrm{Z}$ relation (3.16) holds since the distribution of imaginary points $i \mathrm{Y} \subset i \mathbb{R}$ makes no influence on the logarithmic submeasure. The proof of part (a) is complete. 
In order to prove part (b), it is sufficient to consider the union $W \cup X$ with the above choice of the distribution of real points $X \subset \mathbb{R}$. By $i \mathbb{R}$-Lindelöf condition $(1.20$ for $W$, the union $\mathrm{W} \cup \mathrm{X}$ satisfies Lindelöf condition (3.1) by part [L1] of Proposition 3.1. By Hadamard-Lindelöf theorem 3.1 there exists an EFET $g \neq 0$ with zeroes distribution Zero $_{g}=\mathrm{W} \cup \mathrm{X}$ vanishing on W, for by (3.6) with W instead of Z inequality (3.16) holds. It is obvious that Zero ${ }_{g}=\mathrm{W} \cup \mathrm{X}$ is still asymptotically separated by angles from $i \mathbb{R}$ since this holds both for $\mathrm{W}$ by assumptions and for the distribution of real points $X \subset \mathbb{R}$.

\section{Versions of Theorems 1.1, 1.2 and 2.1 Without CONDition of LOCATION OF DISTRIBUTION OF POINTS W IN RIGHT HALF-PLANE $\mathbb{C}_{\mathrm{rh}}$}

Below we provide a version of Theorem 1.1 with an arbitrary distribution of points $\mathrm{W}$ of a finite upper density.

Theorem 4.1. Condition (1.11) of finiteness of the upper density of $\mathrm{Z}$ and $\mathrm{W}$ is sufficient for each of statements $I$ and III in Theorem 1.1 to be equivalent to a statement:

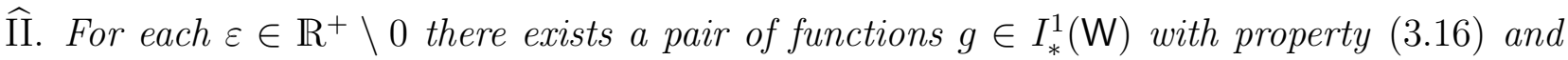
$f \in I_{*}^{1}(\mathrm{Z})$ and a Borel subset $E \subset \mathbb{R}$ satisfying (1.12).

Proof. According to part (a) of Corollary 3.1, there exists an EFET $g \neq 0$ with the properties formulated in Statement $\widehat{\mathrm{II}}$, while by Statement $\mathrm{I}$ in Theorem 1.1 there exists $f \in I_{*}^{1}(\mathrm{Z})$ satisfying 1.12 . This proves the implication $\mathrm{I} \Longrightarrow \widehat{\mathrm{II}}$.

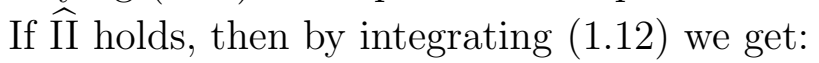

$$
J_{i \mathbb{R}}(r, R ; \ln |f|) \leqslant J_{i \mathbb{R}}(r, R ; \ln |g|)+\varepsilon \ln \frac{R}{r}+C \quad \text { for all } 1 \leqslant r<R<+\infty .
$$

By Lemma 2.1 twice applied respectively to EFET $f \neq 0$ and EFET $g \neq 0$, for some positive numbers $C_{f}, C_{g}, M$ we obtain:

$$
\begin{aligned}
l_{\mathrm{Z}}(r, R) & \stackrel{\sqrt[1.9]{5}}{\leqslant} l_{\text {Zero }_{f}}(r, R) \stackrel{(2.2)}{\leqslant} J_{i \mathbb{R}}(r, R ; \ln |f|)+C_{f} \stackrel{4.17}{\leqslant} J_{i \mathbb{R}}(r, R ; \ln |g|)+\varepsilon \ln \frac{R}{r}+C+C_{f} \\
& \stackrel{2.2 p}{\leqslant} l_{\text {Zero }_{g}}(r, R)+C_{g}+\varepsilon \ln \frac{R}{r}+C+C_{f} \stackrel{\text { 3.16) }}{\leqslant} l_{\mathrm{W}}(r, R)+M+C_{g}+\varepsilon \ln \frac{R}{r}+C+C_{f}
\end{aligned}
$$

for all $1 \leqslant r<R<+\infty$, where we have employed condition (3.16) from II. Redenoting the sum $M+C_{g}+C+C_{f}$ by $C$, we arrive at relation $(1.13)$ in Statement III in Theorem 1.1.

The validity of the implication $\mathrm{III} \Longrightarrow \mathrm{I}$ in Theorem 1.1 has already been mentioned in Remark 2.1 for arbitrary points distributions $\mathbf{Z}$ and $\mathbf{W}$ of a finite upper density.

A version of Theorem 1.2 with $i \mathbb{R}$-Lindelöf condition 1.20 for $\mathrm{W}$ instead of $W \subset \mathbb{C}_{\mathrm{rh}}$ is as follows.

Theorem 4.2. Assume that under (1.11) both $\mathrm{Z}$ and $\mathrm{W}$ are asymptotically separated by angles from $i \mathbb{R}$ in the sense of $(1.15)$ and the distribution of points $\mathbf{W}$ satisfies $i \mathbb{R}$-Lindelöf condition 1.20). Then each of Statements I and III in Theorem 1.2 is equivalent to a statement:

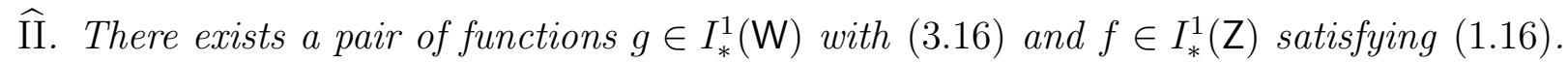

Proof. By part (b) of Corollary 3.1 there exists an EFET $g \neq 0$ with the properties formulated in Statement II, while by Statement II of Theorem 1.2 there exists $f \in I_{*}^{1}(\mathrm{Z})$ satisfying (1.16) and this proves the implication $\mathrm{I} \Longrightarrow \mathrm{II}$.

If Statement II holds, then we integrate as in (2.1), and for each $r_{0} \in \mathbb{R}^{+} \backslash 0$, for some bounded function $Q:\left[r_{0},+\infty\right) \rightarrow \mathbb{R}^{+}$satisfying the condition

$$
\lim _{x \rightarrow+\infty} \frac{Q(x)}{x}=0
$$


and for some number $C_{0} \in \mathbb{R}^{+}$in relation $(1.16)$ we obtain:

$$
J_{i \mathbb{R}}(r, R ; \ln |f|) \leqslant J_{i \mathbb{R}}(r, R ; \ln |g|)+\int_{r}^{R} \frac{Q(y)}{y^{2}} \mathrm{~d} y+C_{0} \quad \text { for all } \quad 0<r_{0} \leqslant r<R<+\infty .
$$

Lemma 4.1 ([12, Cor. 2.1]). Let $r_{0} \in \mathbb{R}^{+} \backslash 0$. If the function

$$
Q:\left[r_{0},+\infty\right) \rightarrow \mathbb{R}^{+}
$$

satisfies 4.2, then there exists a decreasing function $d:\left[r_{0},+\infty\right) \rightarrow \mathbb{R}^{+}$, for which

$$
\begin{aligned}
\int_{r}^{R} \frac{Q(x)}{x^{2}} \mathrm{~d} t & \leqslant d(R) \ln \frac{R}{r} \text { for all } r_{0} \leqslant r<R<+\infty, \\
\lim _{R \rightarrow+\infty} d(R) & =0 .
\end{aligned}
$$

If the function $d:\left[r_{0},+\infty\right) \rightarrow \mathbb{R}^{+}$satisfies (4.5), then there exists an increasing function $Q:\left[r_{0},+\infty\right) \rightarrow \mathbb{R}^{+}$satisfying $(4.2$ and

$$
d(R) \ln \frac{R}{r} \leqslant \int_{r}^{R} \frac{Q(x)}{x^{2}} \mathrm{~d} x \quad \text { for all } \quad r_{0} \leqslant r<R<+\infty .
$$

By the first part of Lemma 4.1, inequality 4.3 can be rewritten as

$$
J_{i \mathbb{R}}(r, R ; \ln |f|) \leqslant J_{i \mathbb{R}}(r, R ; \ln |g|)+d(R) \ln \frac{R}{r}+C_{0} \quad \text { for all } \quad r_{0} \leqslant r<R<+\infty,
$$

where $d$ is some bounded function with property (4.5).

By Lemma 2.1 twice applied respectively to the EFET $f \neq 0$ and to the EFET $g \neq 0$, for some positive numbers $C_{f}, C_{g}, M$ we obtain:

$$
\begin{aligned}
& l_{\mathrm{Z}}(r, R) \stackrel{\sqrt[1.9]{\leqslant}}{\leqslant} l_{\text {Zero }_{f}}(r, R) \stackrel{\sqrt[2.2]{*}}{\leqslant} J_{i \mathbb{R}}(r, R ; \ln |f|)+C_{f} \stackrel{\sqrt[4.7]{\leqslant}}{\leqslant} J_{i \mathbb{R}}(r, R ; \ln |g|)+d(R) \ln \frac{R}{r}+C_{0}+C_{f} \\
& \stackrel{2.2}{\leqslant} l_{\text {Zero }_{g}}(r, R)+C_{g}+d(R) \ln \frac{R}{r}+C_{0}+C_{f} \stackrel{\text { 3.16) }}{\leqslant} l_{\mathrm{W}}(r, R)+M+C_{g}+d(R) \ln \frac{R}{r}+C_{0}+C_{f}
\end{aligned}
$$

for all $r_{0} \leqslant r<R<+\infty$, where we have employed property 3.16 in $\widehat{\text { II. Letting } C:=}$ $M+C_{g}+C_{0}+C_{f}$, we obtain relation (1.17) in Statement III of Theorem 1.2.

The validity of implication $\mathrm{III} \Longrightarrow$ I in Theorem 1.2 has already been mentioned in Remark 2.1 for arbitrary distributions of points $\mathbf{Z}$ and $\mathbf{W}$ of finite upper density.

A version of Theorem 2.1, extending Malliavin-Rubel theorem 1.3 , with $i \mathbb{R}$-Lindelöf condition 1.19) for $\mathrm{W}$ instead of location of $\mathrm{W}$ in the right half-plane reads as follows.

Theorem 4.3. Let the assumptions of Theorem 4.2 be satisfied. Then each of Statements 1 and III in Malliavin-Rubel theorem I.2 is equivalent to the following statement:

II. There exist a pair of functions $g \in I_{*}^{1}(\mathrm{~W})$ with $(3.16)$ anf $f \in I_{*}^{1}(\mathrm{Z})$ satisfying (1.18).

Proof. By part (b) of Corollary 3.1 there exists an EFET $g \neq 0$ with the properties formulated in Statement II, while by Statement II in Malliavin-Rubel theorem 1.2 there exists $f \in I_{*}^{1}(\mathrm{Z})$ satisfying $(1.18)$ and this proves the implication $\mathrm{I} \Longrightarrow \widehat{\mathrm{II}}$.

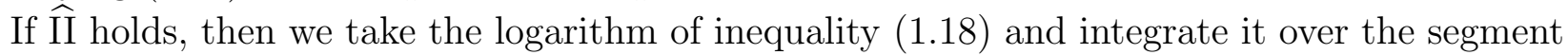
$[r, R]$ and divide as in (2.1). This gives inequalities (2.3) and the passage to (2.4) exactly in the same way as in the proof of the implication $\mathrm{II} \Longrightarrow \mathrm{III}$ in Theorem 2.1. Applying condition 3.16 in Statement $\widehat{\mathrm{II}}$, we can continue (2.4) with some number $C_{0} \in \mathbb{R}^{+}$as

$$
l_{\mathrm{Z}}(r, R) \leqslant l_{\text {Zerog }}(r, R)+C_{g}+C_{f} \stackrel{\sqrt[3.16]{\leqslant}}{\leqslant} l_{\mathrm{W}}(r, R)+C_{0}+C_{g}+C_{f} \quad \text { for all } \quad r_{0} \leqslant r<R<+\infty,
$$

and for $C:=C_{0}+C_{g}+C_{f} \in \mathbb{R}^{+}$this gives III from Malliavin-Rubel theorem 1.2 with 1.19 ).

The validity of the implication III $\Longrightarrow$ Thas already been mentioned in Remark 2.1. 


\section{BIBLIOGRAPHY}

1. P. Malliavin, L.A. Rubel. On small entire functions of exponential type with given zeros // Bull. Soc. Math. France. 89:2, 175-201 (1961).

2. B.N. Khabibullin. Completeness of exponential systems and uniqueness sets. 4th ed. Bashkir State University, Ufa (2012). (in Russian).

3. R.P.Jr. Boas. Entire Functions. Academic Press, New York (1954).

4. B.Ya. Levin. Distribution of zeros of entire functions. Gostekhizdat, Moscow (1956). [Amer. Math. Soc., Providence, RI (1980).]

5. B.Ya. Levin. Lectures on entire functions. Amer. Math. Soc., Providence, RI (1996).

6. L.A. Rubel, J.E. Colliander. Entire and meromorphic functions. Springer-Verlag, New York (1996).

7. B.N. Khabibullin. On the growth of entire functions of exponential type along the imaginary axis // Dokl. AN SSSR. 302:2, 270-273 (1988). [Sov. Math. Dokl. 38:2, 276-278 (1989).]

8. B.N. Khabibullin. On the growth of entire functions of exponential type along the imaginary axis // Matem. Sbornik. 180:5, 706-719 (1989). [Math. USSR-Sbornik. 67:1, 149-163 (1990).]

9. B.N. Khabibullin. On the growth of the entire functions of exponential type with given zeros along a line // Anal. Math. 17:3, 239-256 (1991). (in Russian).

10. B.N. Khabibullin. On the growth of entire functions of exponential type near a straight line // Matem. Zamet. [Math. Notes. 70:4, 560-573 (2001).]

11. B.N. Khabibullin, A.V. Shmeleva. Balayage of measures and subharmonic functions to a system of rays. I: The classical case // Alg. Anal. 31:1, 156-210 (2019). [St. Petersbg. Math. J. 31:1, 117-156 (2020).]

12. A.E. Salimova, B.N. Khabibullin. Distribution of zeros of exponential-type entire functions with constraints on growth along a line // Matem. Zamet. [Math. Notes. 108:4, 579-589 588-600.]

13. B.N. Khabibullin, A.V. Shmeleva, Z.F. Abdullina. Balayage of measures and subharmonic functions to a system of rays. II. Balayages of finite genus and growth regularity on a single ray // Alg. Anal. 32:1, 208-243 (2020). [St. Petersburg Math. J. 32:1, 155-181 (2021).]

Anna Evgenievna Salimova,

Bashkir State Universirty,

Zaki Validi str. 32,

450076, Ufa, Russia

E-mail: anegorova94@bk.ru

Bulat Nurmievich Khabibullin,

Bashkir State Universirty,

Zaki Validi str. 32,

450076, Ufa, Russia

E-mail: khabib-bulat@mail.ru 